Swarthmore College

Works

$2-26-2003$

\title{
Order Parameter Measurements Of Dichroic Dyes Dissolved In Smectic Liquid Crystals That Tilt Without Layer Contraction
}

Peter J. Collings

Swarthmore College, pcollin1@swarthmore.edu

B. R. Ratna

R. Shashidhar

Follow this and additional works at: https://works.swarthmore.edu/fac-physics

Part of the Physics Commons

Let us know how access to these works benefits you

\section{Recommended Citation}

Peter J. Collings, B. R. Ratna, and R. Shashidhar. (2003). "Order Parameter Measurements Of Dichroic Dyes Dissolved In Smectic Liquid Crystals That Tilt Without Layer Contraction". Physical Review E. Volume 67, Issue 2. DOI: 10.1103/PhysRevE.67.021705

https://works.swarthmore.edu/fac-physics/127

This work is brought to you for free by Swarthmore College Libraries' Works. It has been accepted for inclusion in Physics \& Astronomy Faculty Works by an authorized administrator of Works. For more information, please contact myworks@swarthmore.edu. 


\title{
Order parameter measurements of dichroic dyes dissolved in smectic liquid crystals that tilt without layer contraction
}

\author{
Peter J. Collings, ${ }^{1,2}$ B. R. Ratna, ${ }^{1}$ and R. Shashidhar ${ }^{1}$ \\ ${ }^{1}$ Center for Bio/Molecular Science and Engineering, Naval Research Laboratory, Code 6900, 4555 Overlook Avenue, SW, \\ Washington, D.C. 20375 \\ ${ }^{2}$ Department of Physics and Astronomy, Swarthmore College, Swarthmore, Pennsylvania 19081
}

(Received 30 March 2002; published 26 February 2003)

\begin{abstract}
Measurements of the orientational order parameter of dissolved dichroic dyes are reported for two smectic$A$ liquid crystals that tilt in the presence of an in-plane electric field without any decrease in the layer spacing. The dye order parameter is determined by measuring the anisotropic absorption of linearly polarized light. Different dyes are used and measurements are also performed on a smectic liquid crystal that tilts with the expected layer contraction to check how closely the measurements reflect the order parameter of the liquid crystal. The variation of the dye order parameter with electric field is in accordance with the recently proposed model of azimuthal disorder of the tilt angle direction, but the surprising finding is that the local dye orientational order parameter is significantly lower than for the smectic liquid crystal that tilts with the expected layer contraction. This suggests that another mechanism might be contributing significantly to the smectic order, one possibility of which is the tendency for different parts of these siloxane-containing molecules to segregate within each layer. Another possible explanation is that the azimuthal disorder is due to a modulated phase with a high enough density of defects to decrease the value of the local order parameter.
\end{abstract}

DOI: 10.1103/PhysRevE.67.021705

PACS number(s): 61.30.Cz, 61.30.Gd, 64.70.Md

\section{INTRODUCTION}

The field of liquid crystals is one of continuing surprises. One recent example of this concerns the smectic phases of liquid crystals. The molecules in all liquid crystal phases possess long-range orientational order, in that the molecules as they undergo diffusion have a slight preference to orient their long axes along one direction, called the director $\hat{n}$, as opposed to any other direction. Smectic liquid crystals also possess long-range positional order, in that the molecules show a slight preference to form a layered structure as they diffuse about the sample. In the smectic- $A(\mathrm{Sm}-A)$ phase, the director is parallel to the layer normal; in the smectic- $C$ $(\mathrm{Sm}-C)$ phase, the director makes an angle $\theta$ with the layer normal. When a liquid crystal undergoes the transition from the Sm- $A$ to Sm- $C$ phase, the layer spacing decreases by the expected factor of $\cos \theta$ in most materials. The recent surprise is the discovery that some liquid crystals go through the $\mathrm{Sm}-A$ to $\mathrm{Sm}-C$ transition with hardly any layer contraction at all [1]!

This finding is of interest for both scientific and technological reasons. The important scientific question is the identification of the structure of a phase in which the director can tilt away from the layer normal without any layer contraction. On the technological side is the possibility of devices that utilize the large amount of tilt and small amount of birefringence possible in these systems without the problems usually associated with maintaining alignment in the presence of changes in the layer spacing. Recent work has demonstrated that these phases exist in systems of molecules with different molecular structures [1-3], that there is some evidence for a segregation of different parts of the molecule within the layers [4], and that the transition from a nontilted to tilted structure is actually a transition from a state of dis- ordered molecular tilt in random directions to a state of ordered tilt in a single direction [5-8].

In fact, for both scientific and technical reasons, many experiments on these phases have focused on the tilt induced by an electric field in the Sm- $A$ phase as opposed to the tilt that occurs at the Sm- $A$ to $\mathrm{Sm}-C$ transition. This is called the electroclinic effect [9], which only occurs if the molecules are chiral. If the electric field is applied perpendicular to the director in the Sm- $A$ phase, the tilt angle continuously increases as the electric field is increased, with the direction of the tilt being orthogonal to the field. A phenomenological understanding of the electroclinic effect is possible using the Landau theory, in which the free energy is expanded in powers of the tilt angle and the polarization. The tilt angle is linear with the electric field for low values of the field, tending to show a saturation type behavior for high values of the field [9]. In most liquid crystals, the molecules tilt collectively as rigid rods and the layer spacing decreases by the factor of $\cos \theta$. Our present understanding of these new materials is that the molecules are already tilted in the Sm-A phase, but that the direction of the tilt is azimuthally disordered. Thus the average orientation of the molecules is perpendicular to the layers, but locally the molecules have a director tilted with respect to the layer normal. Application of an electric field tends to align the transverse dipole on these molecules, upsetting the random distribution of local directors and producing an average director tilted away from the layer normal. Careful studies of the tilt angle and birefringence behavior as the electric field and temperature are varied confirms this general model of the electroclinic effect in two series of liquid crystals that tilt without layer contraction $[7,8]$.

But the extremely important question of the structure of the phase remains. How is the azimuthal disorder produced 
in the Sm- $A$ phase of these materials? Clearly the regions in which the tilt is azimuthally ordered have dimensions less than the wavelength of light, or else the Sm- $A$ phase would not show a uniform optical axis perpendicular to the layers. Perhaps each layer has a single tilt direction (as in the Sm-C phase), but the tilt direction is azimuthally disordered in going from layer to layer. Perhaps each layer is a dynamic collection of regions each with a single tilt direction. Even more provocative, perhaps there is some type of modulation or spatial periodicity in the azimuthal angle associated with the tilt direction [10]. Recent light scattering data from an azimuthally disordered system provides evidence that seems to point in this direction [11]. Determining whether one of these scenarios, or some other scenario, corresponds to this phase is an active area of investigation.

Only one measurement of the orientational order parameter has been made in these systems and that was in an x-ray experiment [12]. The results of this experiment are consistent with the azimuthal disorder model for these phases and the inferred value of the local orientational order parameter is lower than the typical value for smectic liquid crystals. Measurement of the orientational order parameter of dissolved dyes is a very different probe of the local structure, since the order of the dye can be sensitive to the local order of the liquid crystal in ways not detectable via $\mathrm{x}$-ray experiments. The results of these measurements with dyes closely follow the prediction of the general azimuthal disorder model that successfully explains the tilt angle, birefringence, and x-ray results, but with one very interesting stipulation. In order to explain the dye order parameter measurements, the value of the local orientational order parameter of the dye must be lower than that has been measured or theoretically predicted for a smectic phase, even lower than the value measured by $\mathrm{X}$-ray experiments [12]. Thus the layer structure in these phases might be stabilized by an interaction not present in conventional smectic phases, a good candidate of which is the tendency for these molecules to order in a way that segregates different parts of the molecule. If such a segregation causes one part of the liquid crystal molecule to be less ordered than the rest of the molecule, then association of the dye with the less ordered part could be the reason for the low value of the local dye order parameter. Alternatively, if a modulated phase with a high enough density of defects is present, the lower value of the order parameter for part of the liquid crystal molecule in the neighborhood of the defects might be the reason the dye local order parameter is lower than normal for smectic phases.

\section{THEORY}

The theory involves averaging of the dielectric tensor $\overleftrightarrow{\boldsymbol{\epsilon}}$ due to the azimuthal disorder. We choose to use the dielectric tensor instead of the electric susceptibility tensor for two reasons. First, our development follows that of Ref. [7], which utilizes the dielectric tensor. Second, here we are interested in the properties of the liquid crystal (perhaps slightly modified by a small amount of added dye), whereas later we will turn our attention to the dye molecules themselves. By using the dielectric tensor when discussing the liquid crystal and the electric susceptibility tensor when addressing the dye, possible confusion will be lessened.

Let the local dielectric constant at optical frequencies have principal values $\epsilon_{a}, \epsilon_{b}, \epsilon_{c}$, where the $c$ axis is along the local director $\hat{n}_{l o c}$, the $b$ axis contains the local polarization due to the transverse molecular dipoles, and the $a$ axis is the third axis orthogonal to the other two axes. In order to explain the tilt angle and birefringence data [7], $\epsilon_{a}$ and $\epsilon_{b}$ have to be slightly different, indicating that the local order is slightly biaxial. Clark et al. [8], have shown that an assumption of biaxiality is not necessary if the tilt angle is allowed to depend on the electric field. Both effects are probably present to some degree. We have chosen to use the biaxiality assumption, since it has been shown to describe well the behavior in the systems investigated in this experiment. The length scale associated with this local reference frame is determined by the distance over which the orientation of the molecules is correlated. Thus this length scale varies with temperature, especially as the transition to the $\mathrm{Sm}-C$ phase is approached. Using the estimates of the dipole moment of a correlated group of molecules given in Ref. [7], for the temperature used in this study we estimate that this local reference frame includes about 1000 molecules.

In the laboratory coordinate system, let the dielectric constant at optical frequencies have principal values $\epsilon_{x}, \epsilon_{y}$, and $\epsilon_{z}$, where the $z$ axis lies along the layer normal and the $x$ and $y$ axes are in the plane of the layers.

Let the local $c$ axis be given in the laboratory coordinate system by polar angle $\eta$ and azimuthal angle $\phi$. We can transform the local dielectric tensor into the laboratory coordinate system by a rotation through $\eta$ about the local $b$ axis, followed by a rotation by $\phi$ about the laboratory $z$ axis. In a phase in which the local director is tilted but azimuthally disordered, $\eta$ represents the tilt angle and the local directors are distributed randomly in $\phi$. Thus averaging the transformed dielectric tensor for a uniform distribution in $\phi$ yields the dielectric tensor in the laboratory coordinate system. If an electric field is applied along the $y$ axis, the interaction of the field with the local polarization disturbs the random distribution in favor of one biased toward low values of $\phi$. In order to perform this average, a mean-field distribution function

$$
\rho(\phi)=\rho_{0} \exp \left(E P_{0} \cos \phi / k_{B} T\right)
$$

is used, where $\rho_{0}$ is a normalization factor, $T$ is the temperature, and $P_{0}$ is an effective dipole moment for a correlated group of molecules. It turns out that the averaged dielectric tensor can be expressed through averages of $\cos \phi$ and $\cos ^{2} \phi$, each of which involves modified Bessel functions. The eigenvectors of this averaged dielectric tensor give the principal optical axes of the sample. One eigenvector is along the $y$ axis, and the angle between the other two eigenvectors (the $x^{\prime}$ and $z^{\prime}$ axes) and the $x$ and $z$ axes gives the optical tilt $\theta(E)$. The expression for $\theta(E)$ is

$$
\tan [2 \theta(E)]=\frac{\sin (2 \eta)\langle\cos \phi\rangle}{\cos ^{2} \eta-\sin ^{2} \eta\left\langle\cos ^{2} \phi\right\rangle-\beta\left(1-\left\langle\cos ^{2} \phi\right\rangle\right)},
$$




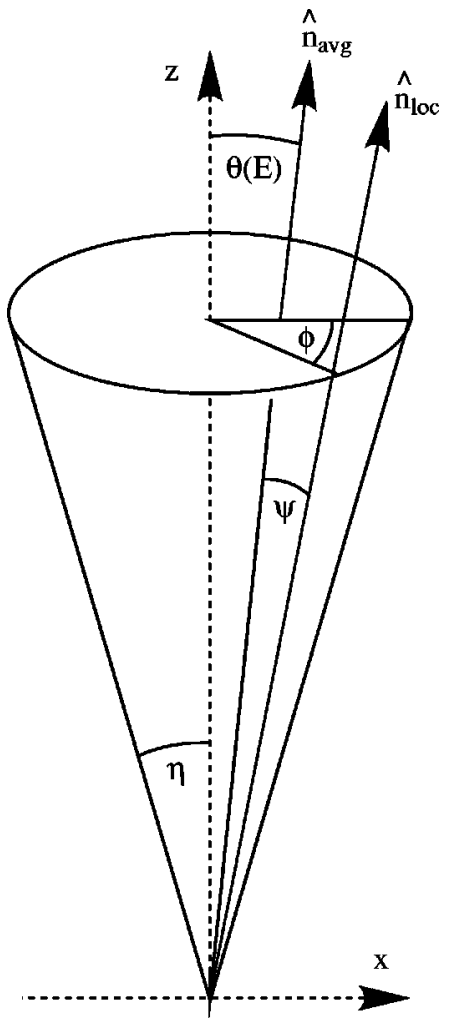

FIG. 1. Relationship between the reference frame for a correlated group of molecules with $\hat{n}_{l o c}$ along the $c$ axis and the reference frame for many such groups with $\hat{n}_{\text {avg }}$ along the $z^{\prime}$ axis. The $z$ axis is normal to the smectic planes and the $x$ axis is in the plane of the layers. For the correlated group of molecules, $\eta$ is the tilt angle and $\phi$ is the azimuthal angle. $\hat{n}_{l o c}$ is given in the reference frame for many correlated groups by polar angle $\psi$ and azimuthal angle $\sigma$ (not shown) relative to the $\left(x^{\prime}, y, z^{\prime}\right)$ reference frame. $\theta(E)$ is the angle between the $z$ axis and $\hat{n}_{\text {avg }}$ ( $z^{\prime}$ axis).

where $\beta$ represents the local biaxiality

$$
\beta=\frac{\epsilon_{b}-\epsilon_{a}}{\epsilon_{c}-\epsilon_{a}}
$$

and the brackets indicate an average using the distribution function of Eq. (1).

The various angles and axes are depicted in Fig. 1. Note that the optical axis making an angle $\theta(E)$ with the $z$ axis is labeled $\hat{n}_{\text {avg }}$, since this is the director one measures for the averaged distribution of local directors. Notice that there are two reference frames under consideration, the local reference frame with $\hat{n}_{l o c}$ along the $c$ axis, and the average reference frame with $\hat{n}_{\text {avg }}$ along the $z^{\prime}$ axis (which is along the $z$ axis when $E=0$ ).

Dichroic dyes can be used to measure order parameters because they absorb light polarized parallel to only one axis of the dye molecule. If this is the long axis of a molecule dissolved in a liquid crystal, the long axis of the dye molecules will align with the director of the liquid crystal. The more the liquid crystal is ordered, the more the dye molecules are ordered and the larger the difference in absorption for light polarized parallel to the director compared to light polarized perpendicular to the director.

One way to understand measurements with dissolved dichroic dyes is to take the absorption of the dye molecules into account through the imaginary part of the electric susceptibility tensor $\overleftrightarrow{\chi}^{i m}$. If it is assumed that the only nonzero element of $\overleftrightarrow{\chi}^{i m}$ in the reference frame of a single dye molecule is the diagonal component representing the long molecular axis, $\chi_{m o l}^{i m}$, then transforming the molecular tensor to the local reference frame in which $\hat{n}_{l o c}$ lies along the $c$ axis yields an imaginary electric susceptibility tensor in the local reference that depends on two order parameters [13]. If the long molecular axis of the the dye is given in the local reference frame by polar angle $\xi$ and azimuthal angle $\zeta$, then the two order parameters are

$$
S=\left\langle\frac{3}{2} \cos ^{2} \xi-\frac{1}{2}\right\rangle, \quad B=\left\langle\sin ^{2} \xi \cos 2 \zeta\right\rangle,
$$

and $\chi_{l o c}^{i m}$ is given by

$$
\chi_{l o c}^{i m}=\left(\begin{array}{ccc}
\frac{1-S}{3}+\frac{B}{2} & 0 & 0 \\
0 & \frac{1-S}{3}-\frac{B}{2} & 0 \\
0 & 0 & \frac{1+2 S}{3}
\end{array}\right) \chi_{m o l}^{i m} .
$$

$S$ is the usual nematic order parameter describing the orientational order of the long axis of the dye molecule. $B$ is a biaxial order parameter describing the tendency of the two short axes of the dye molecule to orient preferentially in directions transverse to $\hat{n}_{l o c}$.

In an absorption experiment, the amount of absorption for light polarized parallel and perpendicular to $\hat{n}_{a v g}$ is what is measured. These are the directions of the eigenvectors of the dielectric tensor described previously. If these directions are denoted by the $x^{\prime}, y$, and $z^{\prime}$ axes (with $\hat{n}_{a v g}$ along $z^{\prime}$ and $\vec{E}$ along $y$ ), then an experiment measures

$$
\begin{aligned}
\frac{\chi_{z^{\prime} z^{\prime}}^{i m}-\chi_{x^{\prime} x^{\prime}}^{i m}}{\chi_{m o l}^{i m}}= & \left\langle\cos ^{2} \psi-\sin ^{2} \psi \cos ^{2} \sigma\right\rangle S \\
& +\left\langle 1+\sin ^{2} \psi-\left(1+\cos ^{2} \psi\right) \cos ^{2} \sigma\right\rangle B,
\end{aligned}
$$

where $\hat{n}_{l o c}$ is given in the primed reference frame by polar angle $\psi$ and azimuthal angle $\sigma$ and the brackets denote an average over $\phi$. For specific values of $\theta(E)$ and $\phi$, the angles $\psi$ and $\sigma$ are given by

$$
\cos \psi=\cos \eta \cos \theta(E)+\sin \eta \sin \theta(E) \cos \phi,
$$

$\sin \psi \cos \sigma=\sin \eta \cos \theta(E) \cos \phi-\cos \eta \sin \theta(E)$.

Both the orientation of the $x^{\prime}$ and $z^{\prime}$ axes and the averages over $\psi$ and $\sigma$ depend on $E$.

The absorptivity is defined as 


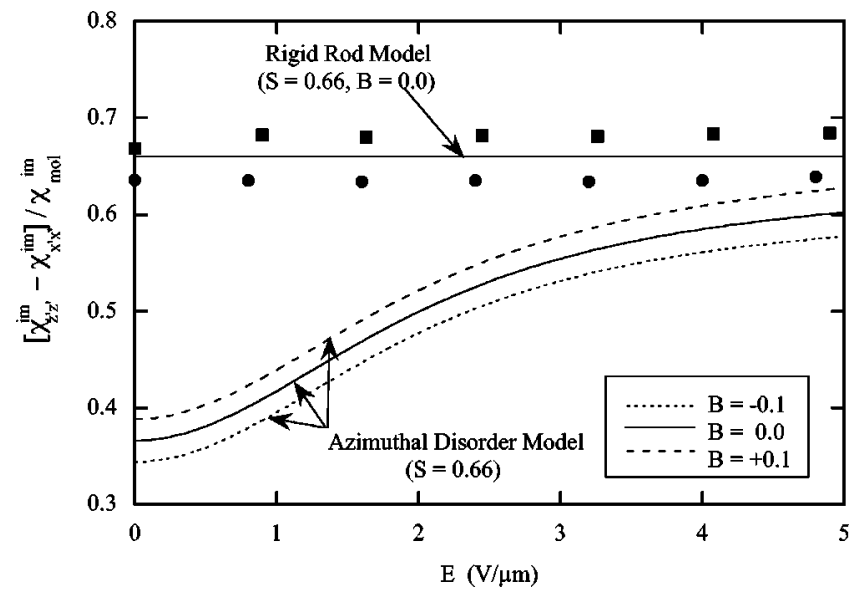

FIG. 2. Predicted variation of $\left[\chi_{z^{\prime} z^{\prime}}^{i m}-\chi_{x^{\prime} x^{\prime}}^{i m}\right] / \chi_{m o l}^{i m}$ with electric field assuming $S=0.66, B=-0.1,0.0$, or +0.1 , and other parameters typical of DSiKN65 $\left(\eta=33^{\circ}, \beta=-0.36, P_{0}=1000 \mathrm{D}, \mathrm{T}\right.$ $=40^{\circ} \mathrm{C}$ ). Also shown are the data for KN125; filled circles, disperse red 1; filled squares, disperse yellow 7 .

$$
\alpha=-\frac{1}{d} \log \left(\frac{I_{\text {out }}}{I_{\text {in }}}\right),
$$

where $d$ is the thickness of the sample and $I_{\text {in }}$ and $I_{\text {out }}$ are the incident and transmitted intensities, respectively. The absorptivity is the quantity measured in an experiment, so one must relate it to the imaginary part of the electric susceptibility. Since the dye molecules are surrounded by a birefringent medium, each diagonal element of the absorptivity tensor is related to the corresponding diagonal element of the imaginary part of the electric susceptibility tensor by [14]

$$
\alpha_{i i}=-k_{0} \chi_{i i}^{i m} / n_{i}
$$

where $n_{i}$ is the index of refraction for light polarized along the direction given by the index $i$ and $k_{0}$ is the wave vector of the light in a vacuum. Thus in terms of the absorptivity measurements, the difference in the two components of the imaginary part of the electric susceptibility tensor is just

$$
\frac{\chi_{z^{\prime} z^{\prime}}^{i m}-\chi_{x^{\prime} x^{\prime}}^{i m}}{\chi_{m o l}^{i m}}=\frac{n_{\|} \alpha_{\|}-n_{\perp} \alpha_{\perp}}{\left[n_{\|} \alpha_{\|}+2 n_{\perp} \alpha_{\perp}\right]_{E=0}}
$$

where the subscripts $\|$ and $\perp$ for $n$ and $\alpha$ indicate indices of refraction and absorptivities measured for light polarized parallel and perpendicular to $\hat{n}_{a v g}$, respectively. It is necessary to use the zero field measurements to determine $\chi_{m o l}^{i m}$ since $\chi_{x x}^{i m}=\chi_{y y}^{i m}$ only when $E=0$.

Figure 2 shows how $\left[\chi_{z^{\prime} z^{\prime}}^{i m}-\chi_{x^{\prime} x^{\prime}}^{i m}\right] / \chi_{m o l}^{i m}$ depends on the electric field assuming $S=0.66, B=-0.1,0.0,+0.1, \eta$ $=33^{\circ}, \beta=-0.36, P_{0}=1000 \mathrm{D}$, and $T=40^{\circ} \mathrm{C}$. Also shown is the line representing the case when there is no azimuthal disorder and the molecules tilt as rigid rods (along with data from KN125, a liquid crystal that has been shown to tilt with the expected decrease in layer thickness). From Eq. (5) it is clear that $\left[\chi_{z^{\prime} z^{\prime}}^{i m}-\chi_{x^{\prime} x^{\prime}}^{i m}\right] / \chi_{m o l}^{i m}$ equals $S-B / 2$ in this case. The order parameter $B$ has not been measured in smectic- $C$
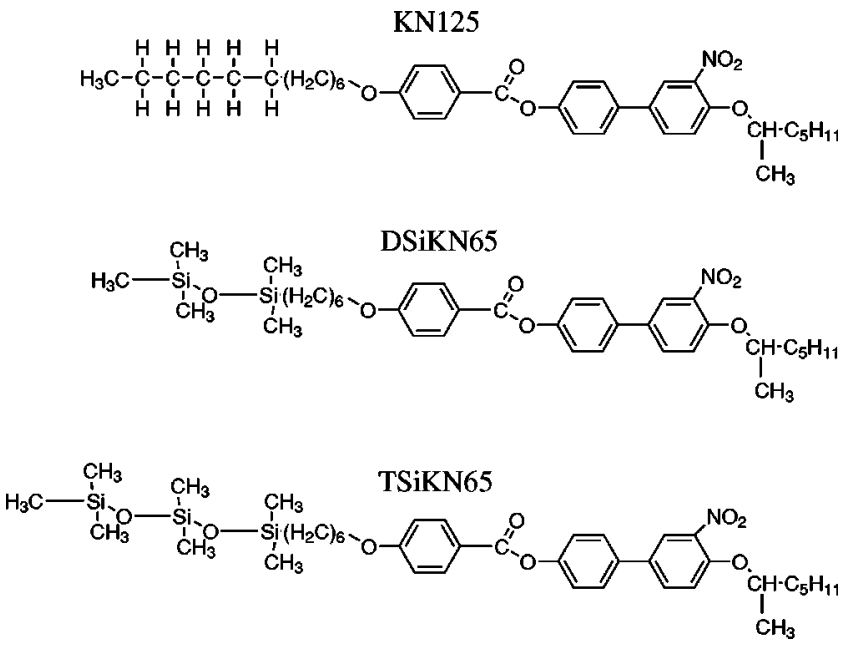

FIG. 3. Smectic liquid crystals investigated in this experiment. The phases and transition temperatures are given in the text.

liquid crystals, but the one measurement of the biaxiality in the refractive indices gives estimates that are extremely small, perhaps 100 times smaller than $S$ [15]. This could explain why the KN125 data does not change much as the electric field, and presumably the biaxiality, increases. Rather large values of $B$ are used in Fig. 2 just in case the biaxiality is significantly larger in liquid crystals that tilt without layer contraction. Note that the curves when azimuthal disorder is present approach the rigid rod line very slowly at high fields.

Since it is difficult to measure $I_{\text {in }}$ and since some intensity can be lost due to reflections at the glass interfaces, it is more practical to make two samples, one of which contains the liquid crystal and the other of which contains the liquid crystal plus a small amount of dye. If the incident light intensity is the same for both samples, then the transmitted light for the liquid crystal sample without dye represents $I_{i n}$ and the transmitted light for the liquid crystal sample with dye represents $I_{\text {out }}$.

Dichroic dyes are routinely used to measure the order parameter of nematic liquid crystals [16], but their use in smectics is much less common. Coles and Gleeson [17] used a single component dye and Coles et al. [18], used a multicomponent dye mixture to measure the order parameter of a Sm- $C$ liquid crystal. Dichroic dyes have also been used to measure the order parameter in the $\mathrm{Sm}-A$ phase of a polymeric liquid crystal [19].

\section{EXPERIMENT}

The liquid crystals used in this investigation are shown in Fig. 3. KN125 represents a conventional smectic liquid crystal in that a tilt in the director away from the layer normal produces a layer contraction with the factor $\cos \theta[20,21]$. $\mathrm{KN} 125$ has a solid-Sm- $A$ transition at $33^{\circ} \mathrm{C}$ and a $\mathrm{Sm}-A$-isotropic transition at $78^{\circ} \mathrm{C}$. DSiKN65 and TSiKN65 represent smectic liquid crystals, in which the director tilts away from the layer normal with only the slightest amount of layer contraction [5]. DSiKN65 has a Sm- $C-\mathrm{Sm}-A$ transition at $39^{\circ} \mathrm{C}$ and a Sm- $A$-isotropic transition at $55^{\circ} \mathrm{C}$. TSiKN65 


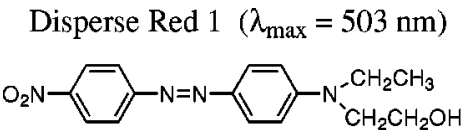

Disperse Yellow $7\left(\lambda_{\max }=388 \mathrm{~nm}\right)$

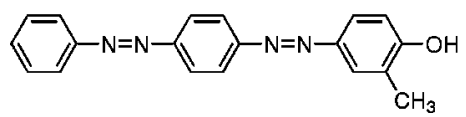

FIG. 4. Dichroic dyes used in this investigation. $\lambda_{\max }$ is the wavelength of maximum absorption.

has a Sm- $C-\mathrm{Sm}-A$ transition at $24{ }^{\circ} \mathrm{C}$ and a $\mathrm{Sm}-A$-isotropic transition at $56^{\circ} \mathrm{C}$.

The dyes used to measure the order parameter of these materials are shown in Fig. 4. We decided to use two different dyes as a check to make sure that none of the results were due to a peculiarity of the dye or liquid crystal. The two dyes differ in the number of aromatic rings and the presence or absence of the $-\mathrm{NO}_{2}$ ligand. The absorption band of disperse red 1 (DR1) peaks at $503 \mathrm{~nm}$ and the measurements were taken using a $488 \mathrm{~nm}$ filter (full-width at half maximum of 3 $\mathrm{nm}$ ). The peak of the absorption band of disperse yellow 7 (DY7) is at $388 \mathrm{~nm}$ and a $436 \mathrm{~nm}$ filter (full-width at half maximum of $9 \mathrm{~nm}$ ) was employed for the measurements. A wavelength longer than the peak absorption wavelength but still well within the absorption band of DY7 was chosen to ensure that little trans-cis isomerization occured with this azo dye.

Two cells were utilized for each liquid crystal-dye combination. In one cell only liquid crystal was used, whereas the other cell contained liquid crystal with between 0.5 and $1.0 \mathrm{wt} \%$ dye. Due to the small amount of dye added to each sample, we could not determine the dye concentration with much accuracy. Each $5 \mu \mathrm{m}$ cell with rubbed polyimide on the surfaces (obtained from E.H.C. Co. Ltd.) was vacuum filled, and the bookshelf geometry was achieved by slowly cooling the sample through the isotropic-Sm- $A$ transition in the presence of a $1 \mathrm{~Hz}$ bipolar square-wave electric field (amplitude of $5 \mathrm{~V} / \mu \mathrm{m}$ ). An Instec $\mathrm{mK}-2$ controller regulated the temperature of the HS-1 hotstage, which was placed on the rotatable stage of a polarizing microscope with a $10 \times$ eyepiece and $5 \times$ objective. The intensity of the transmitted light was measured by a silicon diode detector, amplifier, and oscilloscope. For each liquid crystal, measurements were taken at one temperature with various electric field values applied through a $10 \mathrm{~Hz}$ square wave.

The combination of birefringence and absorption in a sample that is placed between two parallel polarizers is fairly unusual, so it is interesting to calculate what is expected. If the retardation is given by

$$
\delta=\frac{2 \pi \Delta n d}{\lambda},
$$

where $\Delta n$ is the birefringence, $d$ is the thickness, and $\lambda$ is the wavelength of the light in a vacuum, then the intensity transmitted by the sample $I(\gamma)$ when the polarizers make an angle $\gamma$ with the director is

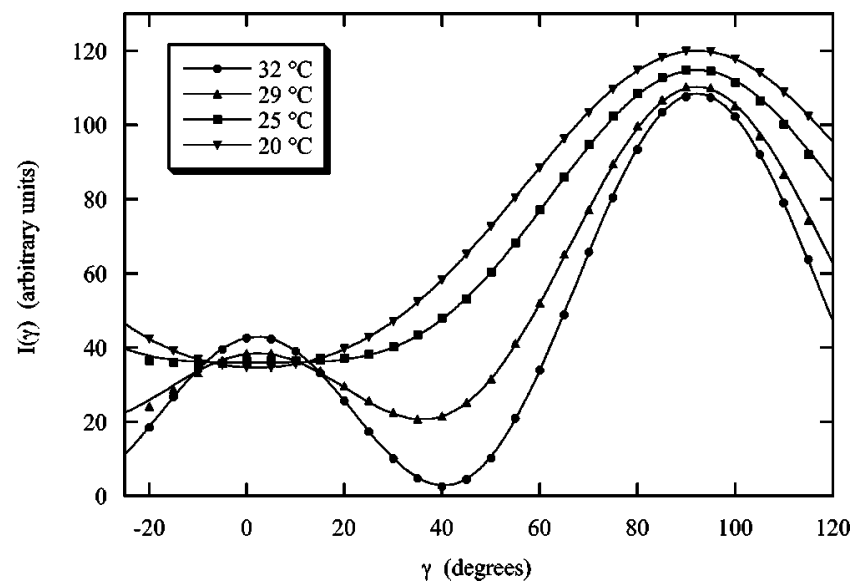

FIG. 5. Transmitted intensity of 5CB/DR1 $(488 \mathrm{~nm})$ at different temperatures as a function of the angle $\gamma$ between the director and the polarizer axis. The lines are fits to Eq. (13).

$$
\begin{aligned}
\frac{I(\gamma)}{I_{0}}= & \cos ^{4} \gamma e^{-2.3 \alpha_{\|} d / 2}+2 \sin ^{2} \gamma \cos ^{2} \gamma e^{-2.3\left(\alpha_{\|}+\alpha_{\perp}\right) d / 2} \cos \delta \\
& +\sin ^{4} \gamma e^{-2.3 \alpha_{\perp} d / 2}
\end{aligned}
$$

where $I_{0}$ is the incident intensity. This expression is similar to the one used in Refs. $[17,18]$ where only one polarizer was employed.

To make sure that the measurement system was working properly, about $1 \mathrm{wt} \%$ of DR1 was dissolved in the nematic liquid crystal 4- $n$-pentyl-4' -cyanobiphenyl (5CB). Varying the temperature of the $5 \mathrm{CB}$ sample changes both the order parameter and the birefringence, thereby allowing us to check our system under a range of experimental conditions. The results of these measurements are shown in Fig. 5, where it can be seen that the birefringence has a strong influence on the shape of the $I(\gamma)$ curve, while the order parameter mainly affects the intensity at the two extrema. Values for the order parameter and birefringence were obtained by fitting the data at each temperature to Eq. (13). These fits are also shown in Fig. 5 and the values resulting from the fits are within $5 \%$ of the literature values for $5 \mathrm{CB}[22,23]$.

\section{RESULTS}

Measurements were taken on samples of KN125 and DSiKN65 each of which had a small amount of either DR1 or DY7 dissolved in it. Measurements were also obtained for a sample of TSiKN65 with dissolved DR1. A single temperature was used for each compound, which in the case of DSiKN65 and TSiKN65 was a temperature in the Sm- $A$ phase but close to the Sm- $C-\mathrm{Sm}-A$ transition. This ensured that there was a significant variation of the tilt angle for modest values of the electric field. The results of all of these measurements are included in Figs. 6 and 7, where the predicted increase in $\left[\chi_{z^{\prime} z^{\prime}}^{i m}-\chi_{x^{\prime} x^{\prime}}^{i m}\right] / \chi_{m o l}^{i m}$ with increasing electric field is clear. For the range of electric field of Figs. 6 and 7, the tilt angle for KN125 varies from zero to about $13^{\circ}$, while for DSiKN65 and TSiKN65 the tilt angle increases from zero to about $30^{\circ}$. 


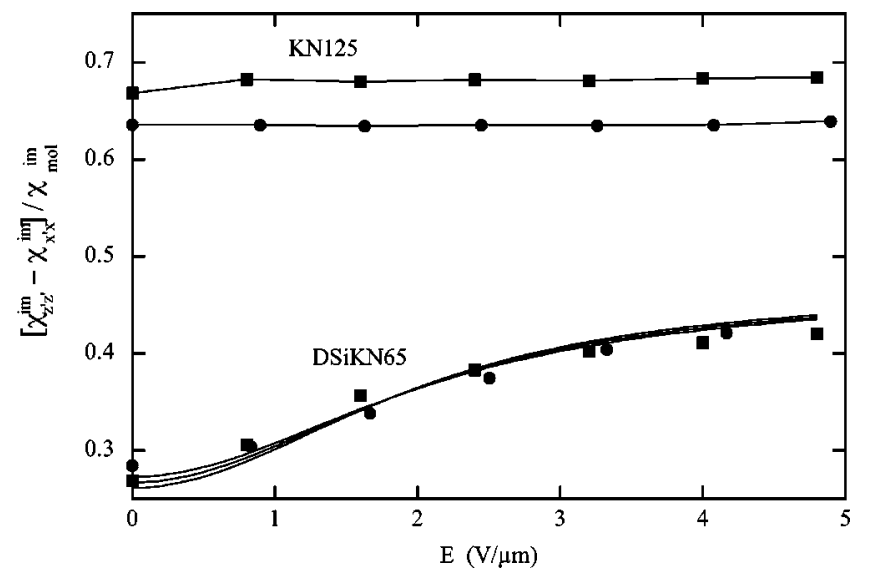

FIG. 6. Values of $\left[\chi_{z^{\prime} z^{\prime}}^{i m}-\chi_{x^{\prime} x^{\prime}}^{i m}\right] / \chi_{m o l}^{i m}$ measured for KN125 $\left(25^{\circ} \mathrm{C}\right)$ and DSiKN65 $\left(40^{\circ} \mathrm{C}\right)$. Filled circles, disperse red 1, filled squares, disperse yellow 7. The lines for the DSiKN65 data are fits to the theory assuming $\eta=33^{\circ}, \beta=-0.36, P_{0}=1000 \mathrm{D}, \mathrm{T}$ $=40^{\circ} \mathrm{C}$, and $B=-0.1,0.0$, or +0.1 . The result of these fits is $S$ $=0.48 \pm 0.04$, where about half of the uncertainty is due to the variation in $B$.

A value for $S$ for the dye molecules in KN125 of about 0.66 (assuming $B \approx 0$ ) is slightly low for a $\mathrm{Sm}-A$ liquid crystal, which may indicate that the dye is slightly less ordered than the liquid crystal molecules. The extremely small change in $S-B / 2$ indicates that significant changes in either $S$ or $B$ do not take place as the electric field is increased, even though the tilt angle increases appreciably.

The behavior of $\left[\chi_{z^{\prime} z^{\prime}}^{i m}-\chi_{x^{\prime} x^{\prime}}^{i m}\right] / \chi_{m o l}^{i m}$ for the dyes in the liquid crystals that tilt with no layer contraction is much different from KN125. The fractional change in this quantity is much larger and the value of this quantity is much lower. While the first finding is exactly what one would expect for a smectic phase with azimuthal disorder, the low value of

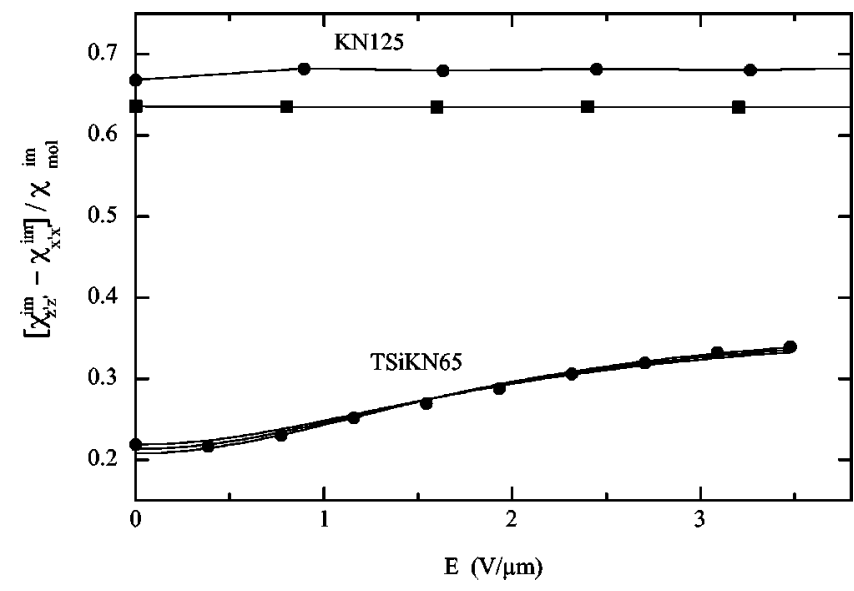

FIG. 7. Values of $\left[\chi_{z^{\prime} z^{\prime}}^{i m}-\chi_{x^{\prime} x^{\prime}}^{i m}\right] / \chi_{m o l}^{i m}$ measured for KN125 $\left(25^{\circ} \mathrm{C}\right)$ and TSiKN65 $\left(25^{\circ} \mathrm{C}\right)$. Filled circles, disperse red 1, filled squares, disperse yellow 7. The lines for the TSiKN65 data are fits to the theory assuming $\eta=33^{\circ}, \beta=-0.36, \quad P_{0}=1000 \mathrm{D}, T$ $=25^{\circ} \mathrm{C}$, and $B=-0.1,0.0$, or +0.1 . The result of these fits is $S$ $=0.38 \pm 0.04$, where about half of the uncertainty is due to the variation in $B$. $\left[\chi_{z^{\prime} z^{\prime}}^{i m}-\chi_{x^{\prime} x^{\prime}}^{i m}\right] / \chi_{m o l}^{i m}$ is a surprise. To be more quantitative, theoretical curves in which the proper azimuthal averages were performed and which used appropriate values for the various parameters [7] were generated. These fits are shown in Figs. 6 and 7, where it can be seen that the best fits yield $S$ values of $0.48 \pm 0.04$ and $0.38 \pm 0.04$ for DSiKN65 and TSiKN65, respectively (where about half of the uncertainty is due to allowing $B$ to vary between -0.1 and +0.1 ). These values for the dye order parameter in a smectic liquid crystal are significantly lower than those predicted for the liquid crystal molecules by theoretical models and also those measured in experiments. For example, McMillan's extension of the Maier-Saupe theory predicts order parameter values in the Sm- $A$ phase higher than about 0.7 [24], and the order parameter values measured using NMR by Doane et al., are also above 0.7 [25]. Either the orientational order of the liquid crystal molecules themselves is much lower than has been seen before, or there is something about the structure of these azimuthally disordered smectic phases that causes the dye molecules to be less ordered than the liquid crystal molecules.

\section{DISCUSSION}

Since the low value of the dye order parameter is the most interesting aspect of the results described in the preceding section, it is worth taking a closer look. It must be kept in mind that it is the order parameter of the dye that is being measured, not the order parameter of the liquid crystal. In many cases when measurements of both have been made, the two order parameters are close in value. This is certainly what we found for DR1 in 5CB. But it is not unusual for the dye to be less ordered than the liquid crystal. For example, we dissolved a small amount of disperse blue 14, an anthroquinone dye, in $5 \mathrm{CB}$ and measured its order parameter at one temperature. Whereas the order parameter of DR1 was 0.52 at that temperature, the order parameter of disperse blue 14 was 0.31 . Clearly the structure of DR1, which resembles the structure of many liquid crystals, is aligned more effectively than the less linear structure of disperse blue 14. Similarly, the proprietary "blue dye" used in Ref. [17], which possesses an absorption spectrum similar to anthraquinone derivatives, yielded a dye order parameter of 0.56 in a Sm-C liquid crystal, while the black dichroic dye used in Ref. [18] gave an order parameter of 0.75 in the same liquid crystal at the same temperature. For these reasons, two dyes with different structures and which yielded order parameters close to the known values in 5CB were used in this experiment. The fact that the results with the two dyes in DSiKN65 are similar argues that there is something different about the local order in DSiKN65 compared to KN125.

If the value of the liquid crystal order parameter is roughly the same in DSiKN65 and TSiKN65 as compared to $\mathrm{KN} 125$, then this should be evident in the birefringence measurements that have been performed on these materials. If the liquid crystal order parameter is similar in all three of these compounds, the similar molecular structures suggest that at high fields (when the azimuthal disorder in DSiKN65 and TSiKN65 is no longer present) the birefringence of all three 


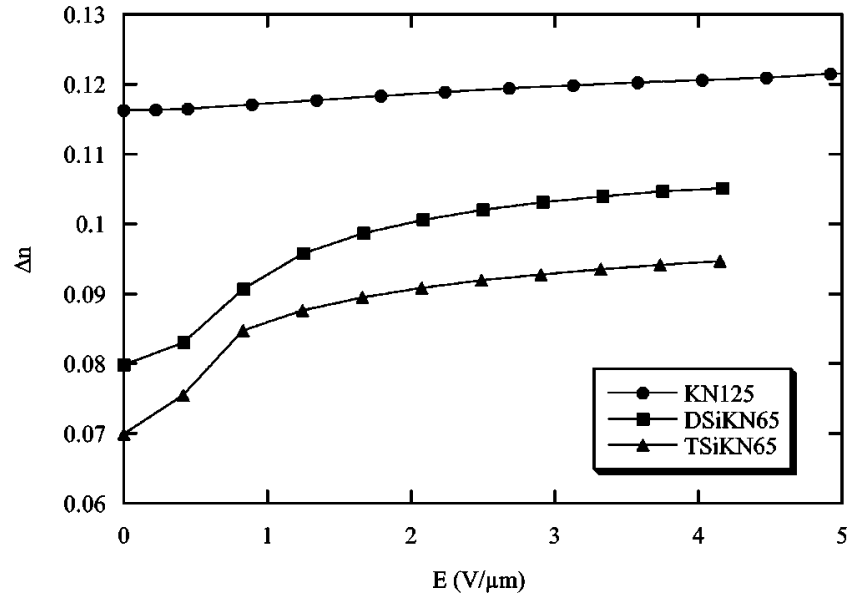

FIG. 8. Birefringence at $633 \mathrm{~nm}$ as a function of electric field for $\mathrm{KN} 125\left(25^{\circ} \mathrm{C}\right)$, DSiKN65 $\left(39.5^{\circ} \mathrm{C}\right)$, and TSiKN65 $\left(24.5^{\circ} \mathrm{C}\right)$.

of these materials should be similar. Prior published data seem to suggest that this is not the case, but it is not entirely unambiguous [6]. As shown in Fig. 8, our measurements are quite convincing in revealing that the birefringence in DSiKN65 and TSiKN65 is significantly lower at high fields than KN125. Note that the ratios of the high field birefringence in these siloxane compounds compared to KN125 (0.88 for DSiKN65 and 0.78 for TSiKN65) are higher than the ratios of the high field dye order parameters of these siloxane compounds relative to KN125 (0.73 for DSiKN65 and 0.58 for TSiKN65), but that in both cases the ratios for TSiKN65 are lower. Thus our finding of low dye order parameters for these $\mathrm{Sm}-A$ phases that tilt without layer contraction probably results from both low order parameters of the liquid crystal molecules themselves and the fact that the dye molecules do not reflect as well the order of the liquid crystal molecules in these materials. If the compounds we studied behave like the compound studied in Ref. [12], then the liquid crystal molecules have an order parameter roughly equal to 0.56 and the dye molecules are more disordered with order parameter values of roughly 0.48 and 0.38 for DSiKN65 and TSiKN65, respectively. In short, while the electric-field dependences of Fig. 8 can be explained by an azimuthal disorder model [6-8], the difference in the high field birefringence cannot be explained by that model. However, this difference can be explained by a lower value for the order parameter.

Since conventional theories of the smectic phase demand that a large value of the orientational order parameter be present to stabilize the phase, one must ask how the smectic phases of these siloxane liquid crystals exist at all if the amount of orientational order is significantly reduced. We do not think that the combination of a lack of layer contraction and a possible low value of the order parameter is a coincidence in these smectic phases. All one needs to do is to refer the arguments put forth in Ref. [4] for the microphase segregation of the siloxane moieties into planes of the layered smectic structure. This segregation into sublayers could then be a type of interaction not present in conventional smectics, but capable of stabilizing smectic phases with smaller amounts of orientational order. Molecular modeling of these liquid crystals indicate that the siloxane chain is not extended, but bends around on itself [5]. If these collapsed siloxane chains tend to aggregate in the same portion of the smectic layer, it is possible that the more linear aromatic and aliphatic parts of the molecules tend to form a tilted structure, but one that is azimuthally disordered. In short, with one end of each molecule "tied down" in the siloxane-rich sublayer, the interactions that would normally cause longrange azimuthal order to be established might not be strong enough to do so. As a result, the linear parts of the molecules are orientationally ordered with tilt, but only short-range order develops in the azimuthal tilt direction. Indeed, Ref. [4] argues on the basis of x-ray and dilatometry measurements that a matching of cross-sectional areas between the aromatic cores and the siloxane end chains is important in stabilizing these phases. It is not difficult to imagine that this interaction, which is a type of excluded volume effect, is strong enough to establish tilt, but not strong enough to produce long-range azimuthal order of the tilt direction. These authors argue that the two diffuse bands seen in x-ray experiments could easily be due to the different correlation lengths of the siloxane chains versus the rest of the molecules.

A completely different model of this phase that is also consistent with lower order parameter measurements is one in which the azimuthal order is due to a modulation of the tilt angle direction in a phase with a high density of defects. For example, Meyer and Pelcovits [10] have proposed a structure with an ordered array of disclination lines and walls. The characteristic length of the proposed structure is estimated to be about 40 molecular spacings, which is small enough not to be seen optically, but large enough to conceivably lower the value of the local order parameter.

Finally, it should be pointed out that both of these types of dye molecules are likely to associate with the more linear and aromatic part of the DSiKN65 and TSiKN65 molecules. Therefore, the order parameter measured in these experiments probably depends more strongly on the order of this part of the liquid crystal molecules as opposed to the siloxane-containing part. The birefringence results are also more sensitive to the linear, aromatic part of the molecules, since this part contributes most strongly to the optical properties in general. So the difference in the amount of orientational order between KN125 and the two siloxane-containing liquid crystals could very well be due to the fact that on average the hydrocardon chain in KN125 is extended and is ordered much like the rest of the molecule, while in DSiKN65 and TSiKN65 the siloxane chain is not extended and is ordered quite differently from the rest of the molecule. This is a more likely scenario if the two parts of the molecules are segregated in each layer, but it is not impossible that this could occur without such segregation.

\section{CONCLUSIONS}

Order parameter measurements of dissolved dichroic dyes are consistent with the present model of $\mathrm{Sm}-A$ phases that tilt with no layer contraction. This is a model involving azimuthal disorder in the tilt direction, which becomes ordered when an electric field is applied or when the transition to the 
Sm- $C$ phase occurs. In addition, these measurements reveal the surprising finding that the amount of dye orientational order (and possibly liquid crystal orientational order) is lower than in smectic liquid crystals with layers that contract when the molecules tilt. One structure that might be consistent with these results for a Sm- $A$ phase with layers that do not contract upon tilting is one in which the siloxane moieties tend to form sublayers that stablize the phase and cause tilt away from the layer normal, but do not produce longrange azimuthal order of the tilt direction. If these materials possess this structure, then they are nature's compromise between smectic liquid crystals and the lamellar phases of amphiphilic molecules. On the order hand, a structure in which the smectic order is broken by an array of defects is also consistent with the experimental results presented here. If this is the case, then there is much to be learned in trying to understand these systems at a fundamental level.

\section{ACKNOWLEDGMENTS}

We would like to thank B. T. Weslowski for helping with the experiments and J. Naciri for synthesizing the liquid crystals. Valuable discussions with M. S. Spector, J. V. Selinger, and D. K. Shenoy are also gratefully acknowledged. This research was supported by the Office of Naval Research and the Naval Research Laboratory.
[1] J. Naciri, J. Ruth, G. Crawford, R. Shashidhar, and B.R. Ratna, Chem. Mater. 7, 1397 (1995).

[2] M.D. Radcliffe, M.L. Brostrom, K.A. Epstein, A.G. Rappaport, B.N. Tomas, R. Shao, and N.A. Clark, Liq. Cryst. 26, 789 (1999).

[3] F. Giesselmann, P. Zugenmaier, I. Dierking, S.T. Lagerwall, B. Stebler, M. Kaspar, V. Hamplova, and M. Glogarova, Phys. Rev. E 60, 598 (1999).

[4] E. Corsellis, D. Guillon, H. Kloess, and H. Coles, Liq. Cryst. 23, 235 (1997).

[5] M.S. Spector, P.A. Heiney, J. Naciri, B.T. Weslowski, D.B. Holt, and R. Shashidhar, Phys. Rev. E 61, 1579 (2000).

[6] J.R. Lindle, F.J. Bartoli, S.R. Flom, J.V. Selinger, R. Shashidhar, and B.R. Ratna, in Liquid Crystals Materials and Devices, edited by T.J. Bunning, S.H. Chen, L.C. Chein, S.-C.A. Lien, T. Kajiyama, and N. Koide, Mater. Res. Soc. Symp. Proc. 559 (Material Research Society, Pittsburgh, 1999).

[7] J.V. Selinger, P.J. Collings, and R. Shashidhar, Phys. Rev. E 64, 061705 (2001).

[8] N.A. Clark et al., Appl. Phys. Lett. 80, 4097 (2002).

[9] R.B. Meyer, Mol. Cryst. Liq. Cryst. 40, 33 (1977).

[10] R.B. Meyer and R.A. Pelcovits, Phys. Rev. E 65, 061704 (2002).

[11] A. Tang, D. Konovalov, J. Naciri, B.R. Ratna, and S. Sprunt, Phys. Rev. E 65, 010703(R) (2001).
[12] J.P.F. Lagerwall, F. Giesselmann, and M.D. Radcliffe, Phys. Rev. E 66, 031703 (2002).

[13] T.E. Lockhart, D.W. Allender, E. Gelerinter, and D.L. Johnson, Phys. Rev. A 20, 1655 (1979).

[14] B.E.A. Saleh and M.C. Teich, Fundamentals of Photonics (Wiley, New York, 1991).

[15] T.E. Lockhart, E. Gelerinter, and M.E. Neubert, Phys. Rev. A 25, 2262 (1982).

[16] B. Bahadur, Handbook of Liquid Crystals (Wiley-VCH, Weinheim, 1998), Vol. 2A.

[17] H.J. Coles and H.F. Gleeson, Mol. Cryst. Liq. Cryst. Lett. 6, 53 (1998).

[18] H.J. Coles, H.F. Gleeson, and J.S. Kang, Liq. Cryst. 5, 1243 (1989).

[19] E. Wolarz and D. Bauman, Liq. Cryst. 19, 221 (1995).

[20] G.P. Crawford, R.E. Geer, J. Naciri, R. Shashidhar, and B.R. Ratna, Appl. Phys. Lett. 65, 2937 (1994).

[21] R.E. Geer, S.J. Singer, J.V. Selinger, B.R. Ratna, and R. Shashidhar, Phys. Rev. E 57, 3059 (1998).

[22] N.V. Madhusudana and R. Pratibha, Mol. Cryst. Liq. Cryst. 89, 249 (1982).

[23] H.J. Coles, in The Optics of Thermotropic Liquid Crystals, edited by S. Elston and R. Sambles (Taylor \& Francis, London, 1998).

[24] W.L. McMillan, Phys. Rev. A 4, 1238 (1971).

[25] J.W. Doane, R.S. Parker, B. Cvikl, D.L. Johnson, and D.L. Fishel, Phys. Rev. Lett. 28, 1694 (1972). 